\title{
Analysis on the Connotation of Probability in the Artificial Intelligence Algorithm for Economic Decision Making
}

\author{
Long Wang \\ Wanglong59@163.com \\ Computing Research Lab, School of Economic \\ Management \\ Shanghai University of Political Science and Law \\ 201701, China
}

\author{
Zhe Wang \\ z.wang2@napier.ac.uk \\ School of Computing \\ Edinburgh Napier University, EH10 5DT,United \\ Kingdom
}

\begin{abstract}
This paper analyzes the inner relations between Classical sub-scheme probability and statistic probability, subjective probability and objective probability, prior probability and posterior probability, Transition probability and probability of utility, and further analysis the goal, method, and its practical economic purpose which represent by these various probability from the perspective of mathematics, so as to deeply understand there connotation and its relation with economic decision making, thus will pave the route for scientific predication and decision making.
\end{abstract}

Keywords-Probability, Benefit, Decision, Analysis

\section{INTRODUCTION}

In the economic prediction and decision making process, what should be decided in advanced by the economic manager is probability. How to define various concepts of probability reasonably and scientifically under different economic situations, and give these probability the practical economic meaning is the start of predication and the key point of decision making, even it is the difficulty of holding the objective law of economic activities scientifically.

\section{Classical Probability and Statistical Probability Classical Probability}

We all know that, in the beginning of the development of the probability theory, the following two objects are recognized as the main research object, which are the random phenomenons belongs to two simple properties.

$$
S=\left\{e_{1}, e_{2}, \cdots, e_{n}\right\}
$$

The elements of sample place of experiments is limited, which is $S=\left\{e_{1}, e_{2}, \cdots, e_{n}\right\}$

(1) the happening of the possibility of each basic event is

equal,so as

$$
P\left(e_{1}\right)=P\left(e_{2}\right)=\cdots=P\left(e_{n}\right)=\frac{1}{n}
$$

generally ,the mathematics model of these types of random experiments is called equal possible probability, the classical probability, in this probability type, the definition on the probability of the happening of the random event $\mathrm{A}$ is: $P(A)=\frac{\text { BasicEventsContainedbyA }}{\text { TheTotalNumberofEventsofSampleSpace }}$

\section{Statistic Probability}

Assume that repeat the same experiment $\mathrm{n}$ times in the random experiment $\mathrm{E}$, if the event $\mathrm{A}$ has occurs $n_{A}$ times, then the ratio $f_{n}(A)=\frac{n_{A}}{n}$ is the frequency of the happening of event $A$, obviously, there are $0 \leq f_{n}(A) \leq 1, f_{n}(S)=1$.

With the growing experiments number of $n$, the frequency $f_{n}(A)$ will be stability, that means it will be stable to a constant, that kinds of " stability of frequency" is the discipline of statistics. So that, the happening probability of the random event can be represent by a variable, this variable will describe the happening probability of the event $\mathrm{A}$ in the experiment, the variable in the rang from 0 to 1 is defined as the probability of $\mathrm{A}$, which represent as $P(A)$, this is the statistic meaning of probability.

Here must be cautious :

(1) although there are existing tightness connections between classical sub-scheme probability definition and the statistic definition of probability, and there are consistent conclusion but it still define the possibility from different routes for the probability of random event in the random experiment. so that, there character must be cautious in the economic activity based on the object there are focusing on.

(2) in the economic predication and decision, it may repeated to use the statistic meaning of probability, for the reason when the experiments number $\mathrm{n}$ is approaching a big value, $f_{n}(A) \approx P(A)$, it must not neglect the equal possibility of the classical probability, such ideal model is widely used, for example, some nature phenomenal cannot be predicated when happening. 


\section{SUBJECTIVE PROBABILITY AND OBJECTIVE PROBABILITY}

The subject probability is the measurement based on the subjective judgment on the possibility of the event based on several experiments people have done. It is the predication on the probability based on experience and presentiment. Obviously, it must obey the axiomatization definition on the probability. that is:

$$
0 \leq P_{i} \leq 1(i=1,2, \cdots \cdots) \sum P_{i}=1
$$

From the personal perspective, the subjective probability is based on several specific experience and specific outcome for personal belief, so that, the personal subjective probability is based on personal knowledge level, working experience, personal quality, judgment ability, all these related factors, etc.Because the subject probability is based on the personal subjective judgment, so that, when people is define the subject probability, there are two distinguished feature:

(1) the difference of the evaluation on the value of the probability: different people may have different value on the probability evaluation under the same condition for the same event; even the same person can also have different value on the probability evaluation under the different time for the same event.

(2) the character that cannot be checked: the subjective probability cannot be checked whether it is correct or not, for example, a manager expected that the profits for next month will be enhance by $80 \%$ probability, but another manger declare that only $50 \%$ probability for enhance the profits, so that,we can not expect who is right, even in the next month, the profit is do enhanced, we can only declare that the probability for enhance the profits at $50 \%$ is wrong.

The objective probability is statistics based on the event developing, for example, based on the statistics in the previous 30 years, we can predict that the snows days in some area is 36 days, based on the statistics mean in probability, the snows probability is $0.10-0.20$.

the Different and Correlation Between Subjective Probability and Objective Probability

The different between subjective probability and objective probability is there extent on reflecting the real facts, but these facts are all based on the relative meaning, because we can not directly compute or statistically compute the absolute probability value for the practical situation. the reason is:

(1)the constrains on the statistics: even the probability is based on the previous huge data and statistics, but this "huge" is still based on the relative constrains, because any " huge" can contain all the factors that reflects all the real practical meanings.

(2)The various of times changing.: one aspects: the data and statistics that used are all pasted materials, another aspects, the markets is always changing dynamically, the past materials can only reflects the past, it can not reflect the current moment and the future, we can only predict the future based on the summarizing and concluding the previous changing rules.

Although, many experts think that, the subjective probability and the objective probability are two organic portion of the degree of uncertain from small to big, they are all non-neglect.
So that, the evaluation of the subjective probability has wide applying prospect in the economic prediction and decision.

\section{THE PRIOR PROBABILITY AND THE POSTERIOR PROBABILITY}

\section{the Prior Probability and the Posterior Probability}

The prior probability, simplified specification: it is based on the historical material or objective judgment to define the probability, the "prior" means the experiment that haven't been verified. Generally, in the common economics decision, the intuition of the experience, subjective judgment and the current situation are all based on the prior probability representation.

For the reason these probability has not been verified by effective methods, there are risk existing. In order to reduce the risk, it needs to precisely hold and evaluate these prior probability. This is based on scientific experiment, investigation, statistics analysis to get these intelligent information, so as to amend these prior probability, so as to enhance the correctness of the probability to the real situation, these probability which has been viewed and further added information is posterior probability.

Obviously, the difference between the prior and posterior probability is:

(1)based on the time line, through the experiment, investigator and various kinds of method to further add information as the boundary, before this boundary or after this boundary.

(2)based on the matching of the real practical situation, the latter is more close to the real situation than the former, it is the modified probability.

2 . the computation on the posterior probability

Generally speaking, the posterior probability is computed based on the experiments and the Bayesian theory, so that, it must be cleared on the following concepts:

$A$, and B are two events in the Sample Space S, we called $P\left(A_{1} / B\right)=\frac{P\left(A_{1} B\right)}{P(B)}$

A under the condition $\mathrm{B}$. as the happening probability of event

(2) based on the above formula the united probability is $P\left(A_{1} B\right)=P\left(A_{1}\right) P\left(B / A_{1}\right)$

(3) Suppose $A_{1} \cup A_{2}=S$ then $P(B)=P\left(S \quad B=P\left(A_{1} B\right)+P\left(A_{2} B\right)=P\left(A_{1}\right) P\left(B / A_{1}\right)+P\left(A_{2}\right) P\left(B / A_{2}\right)\right.$

is the marginal probability

Following is an practical example;

Some company needs to choose the salesman, in order to find the most qualified salesman, following steps are implemented. First, define the prior probability: collect ,organize and analysis the previous materials, the outcome is: in all these salesman who submit their application, only $63 \%$ of them are qualified for the practical requirements, the rest of them are not qualified, $63 \%$ is the prior probability. 
Secondly, using the effective method to capture the precise information( such as experiment): holding the " sale ability testing", the outcome is: in these qualified salesman, only $82 \%$ of them can pass the testing, only $28 \%$ of those non-qualified salesman pass the testing.

The third steps: computing and modify the prior probability( posterior probability): using $A_{1}$ as the representation of the qualified salesman, and using B as passing the testing, then;

conditional probability $P\left(B / A_{1}\right)=0.82$

united probability

$P\left(A_{1} B\right)=P\left(A_{1}\right) P\left(B / A_{1}\right)=0.63 \times 0.82=0.516$

Marginal probability

$P(B)=P\left(A_{1}\right) P\left(B / A_{1}\right)+P\left(A_{2}\right) P\left(B / A_{2}\right)=0.516+0.131=0.647$

so that

$P\left(A_{1} / B\right)=\frac{P\left(A_{1}\right) P\left(B / A_{1}\right)}{P\left(A_{1}\right) P\left(B / A_{1}\right)+P\left(A_{2}\right) P\left(B / A_{2}\right)} \approx \frac{0.516}{0.647} \approx 0.8$

this example shws that:

(1) using the prior $P\left(A_{1}\right)$, and then get information $P\left(B / A_{1}\right)$, so as to compute $P\left(A_{1} / B\right)$

(2) through computing we found: $P\left(A_{1}\right)=0.63=63 \%$ raise to $P\left(A_{1} / B\right)=0.8=80 \%$

(3) holding the "ability testing" is valuable, so as to present that the inner meaning of the posterior probability

Above all, compute the posterior has the following function

(1) if the information proposed is non-complete information or subjective probability, so that, through the Bayesian theory to compute the posterior probability to provide a further research scientific method, and can do quantification on the result for its possibility.

(2)if any investigation result cannot be absolutely correct, and the prior knowledge or subjective probability are not believable, then the computed posterior probability can combine these two information in a skillfully manner.

(3) it can be repeated used in economic predication and decision process, it makes the decision more reasonable and scientific.

\section{TRANSITION Probability AND UTILITy ProbabiLITY}

\section{In the risk type decision making,}

There are various standard that can be choose for decision making, the most common used methods are: using the expected valued as the standard, using classical probability as the standard and using the maximum value as the standard,etc.but whatever choose what kind of standard, the initial question is the value problem of the probability, generally speaking, it needs to be classified into two layers for discussing.
In the first layer, based on various practical situation of the probability for the nature situation: if the occurs of the probability has the obvious objective character and stabilize, then choose expected value as the standard; if various kinds of nature situation cannot be predicted for its probability, then choose equal probability as the standard; if some particular situations' probability is obvious higher than the other probability of the plans, and these expected value has not so much different, then choose the maximum possibility as the standard.

In the second layer, based on the previous experience and decided probability and its future changing possibility: whatever which standard choose for the standard for decision, the pre-requirement is define the occur probability for various situations, however, these probability are evaluated based on the past experience, but the decision is for the future, so that, once the value of the probability has changed, then will the decision plan still correct?

So that, we use the method of reverse thinking, exploring that how much affect it will taken as the changing of the current decided probability based on previous experience, and how it will threat to the current optimized plan? In other words, we need to find such rang that for the various changing of the probability, only if the probability is inside the rang of the probability, this optimized plan will be still effective, if not, the plan should be adjusted, however, the endpoint of the rang is the critical point, the probability that correspond to the critical point is the transition probability.

\section{2. the computation of the transition probability}

If use the expectation as the standard, then the new invested products is the most optimized plan, if the future probability may has large change, then we must compute the transition probability $\mathrm{P}$.

$\mathrm{P}=0.5$

Use $\mathrm{P} \times 8+(1-\mathrm{P}) \times(-2)=\mathrm{P} \times(-3)+(1-\mathrm{P}) \times 9$ to get $\mathrm{P}=0.5$

Only if $\mathrm{P}>0.5$, then the new invest products is still the most optimized plan; only if $\mathrm{p}<0.5$, then just the opposite plan, only if $p=0.5$ then two of them has the same profits.

\section{3. the propose of the utility probability}

(1) utility

Let see an example: there are two solutions. The solution one get 200 profit by 0.5 probability, and lose 100 by 0.5 probability; the solution two get 20 by $100 \%$, obviously, the solution one get 50 expected value which is far more big than the 20 expected value of solution two, but under the practical decision situation, most of people will choose solution two so as to avoid the risk of lose. Of course, some of them would like to choose solution one. We can see from this example:

(1)The economic decision is not he "mechanical decision", in the process of decision, mental factors, subjective initiative has 
the dominate position, because the decision is made by people. The experience, intelligent, power and judgment ability ,such these subjective initiative factors must make important affect on the decision.

(2) the conservative decision people has different action with the aggressive people when doing the same expected value.

(3) in the previous decision methods, most of them using direct computing( such as expected value) as the decision standard, this is obviously has more constrains in practical situation.

\section{(2) Utility curve}

Generally Speaking, when the same decision has multiples or low risk of losing, the decision makers' interests and expected value has high degree in matching; when the decision has only once chance, and the risk is high, the decision makers' interests and expected value has low matching. Above all, the decision maker have different utility value for the same profit of various risks.

Above all, if we use abscissa represent profit, ordinate as utility value, then the changing relation of the attitude on the risk can be draw as a curve. Then we call this curve as the decision makers utility curve, and the utility (function) can be represent by this curve.

\section{(3) utility probability}

We conclude: the utility value is represented as probability, $\mathrm{P}, 0 \leq \mathrm{P} \leq 1$, maximum profit of the utility value is 1 , minimum profit of the utility value is 0 .

\section{4. the definition of the utility probability}

Just as described above, utility is used to identify the attitude of risk for the decision maker, so as to say that utility is the subjective measurement for the decision maker.

So that, we can using mental testing method to define the utility value. For example, the decision maker will accept definitive 20 profit and won't accept solution one, this indicate that 20 profit's affection is bigger than solution one, more question, how about 5 profit for solution two? If the decision maker accept 5 profit solution, then it indicate that the utility of 5 profit is bigger than the utility of solution one, more questions, if the profit lose 5 for solution two? If the decision maker still accept solution one, then its indicate solution one has more utility value than 5 profit.

Thus, with continuously changing of the getting profit, the decision maker will finally find the same effect between the profit and loss value and the solutions, we suppose the profit and the loss value is 0 ,then it will be equal with the 0 corresponding with the utility value and the solution one utility value $0.5 \times 1.0+0.5 \times 0=0.5$, so as to get the 0 profit and the loss value is 0.5 , the point on the curve is $(0,0.5)$.
With the same method, if get 200 profit by 0.5 probability, the profit is 0 with the 0.5 probability, repeat the above process, suppose the 60 utility has the same utility with the above solution, the profit and loss value for 60 is $0.5 \times 1+0.5 \times 0.5=0.75$, so that we can get the point on the curve $(60,0.75)$.

By continuously using the same method can get more points on the curve, by connecting these points can get the utility curve for the decision maker.

On the opposite, by getting the profit value, using utility curve can get the utility value. Something needs to be focus on: although the transition probability and the utility probability are two important probability concept in the risk decision process, but these two are different. The utility probability ( utility value) is used to describe the attitude for the risk of the decision maker, it is subjective probability, but the transition probability is objective probability, it is correspond to the nature probability as the probability on the critical point, it has obvious character of objective.

\section{CONCLUSION}

1 Based on the analysis of classical probability and statistical probability, subjective probability and objective probability, prior probability and posterior probability, transition probability and utility probability, it is not hard to find on the precisely define various concepts of probability, fully advantage the math function can provide effective service for economic decision support.

2 With the high speed developing of economics, the real economic decision activity provide objective condition for various real concepts of probability, at the same time, not only economics or management has propose high demand for criteria and standard.

\section{References}

[1] G. Eason, B. Noble, and I.N. Sneddon, "On certain integrals of Lipschitz-Hankel type involving products of Bessel functions," Phil. Trans. Roy. Soc. London, vol. A247, pp. 529-551, April 1955. (references)

[2] J. Clerk Maxwell, A Treatise on Electricity and Magnetism, 3rd ed., vol. 2. Oxford: Clarendon, 1892, pp.68-73.

[3] I.S. Jacobs and C.P. Bean, "Fine particles, thin films and exchange anisotropy," in Magnetism, vol. III, G.T. Rado and H. Suhl, Eds. New York: Academic, 1963, pp. 271-350.

[4] K. Elissa, "Title of paper if known," unpublished.

[5] R. Nicole, "Title of paper with only first word capitalized," J. Name Stand. Abbrev., in press.

[6] Y. Yorozu, M. Hirano, K. Oka, and Y. Tagawa, "Electron spectroscopy studies on magneto-optical media and plastic substrate interface," IEEE Transl. J. Magn. Japan, vol. 2, pp. 740-741, August 1987 [Digests 9th Annual Conf. Magnetics Japan, p. 301, 1982].

[7] M. Young, The Technical Writer's Handbook. Mill Valley, CA: University Science, 1989. 